\title{
Efectos de la introducción progresiva de los inhibidores de la calcineurina en la función renal de una cohorte de pacientes que recibieron trasplante de hígado
}

\section{Effects of progressive introduction of calcineurin inhibitors on the renal function of a cohort of liver transplant recipients}

\author{
Yeinis Paola Espinoza, Juan Carlos Restrepo, John Jairo Zuleta, \\ Juan Ignacio Marín, Octavio Germán Muñoz, Sergio Iván Hoyos, \\ Carlos Guzmán, Álvaro Mena, Gonzalo Correa - Medellín (Colombia)
}

\section{Resumen}

Introducción: la enfermedad renal aguda (ERA) se presenta en el postrasplante de hígado con una incidencia que varía de $12-64 \%$, con una mortalidad intrahospitalaria asociada de $40-67 \%$. Este estudio utiliza los criterios RIFLE para analizar el efecto del esquema inmunosupresor con inhibidores de la calcineurina en la aparición de ERA en los primeros quince días postrasplante de hígado. También evalúa el protocolo de introducción tardía y progresiva de los medicamentos inhibidores de la calcineurina (CNi) como estrategia para disminuir la incidencia de ERA postrasplante.

Métodos: estudio analítico de cohortes de 163 pacientes con trasplante de hígado.

Resultados: ciento sesenta y tres pacientes cumplieron con los criterios de inclusión del estudio con un promedio de edad de 51 (46-56) años. De éstos, sólo 11 (6.74\%) presentaron ERA postrasplante. Ciclosporina fue administrada a $126(77.3 \%)$ de los pacientes trasplantados y tacrolimus a 21 (12.88\%). Inmediatamente después del trasplante los pacientes fueron clasificados en dos grupos: pacientes con riesgo alto de desarrollar ERA y pacientes sin problemas renales. A los primeros se les inició el CNi a partir del tercer día postrasplante y a los últimos entre seis y 18 horas después del trasplante, a una dosis que se aumentó gradualmente

Conclusiones: la introducción tardía y progresiva de los CNi podría ser una estrategia efectiva para disminuir la incidencia de ERA en el postrasplante de hígado (Acta Med Colomb 2011; 36: 130-134).

Palabras clave: trasplante hepático, enfermedad renal aguda, criterios RIFLE, inhibidores de la calcineurina

\section{Abstract}

Background: acute renal disease (ARD) occurs in liver transplantation with an incidence ranging from 12 to $64 \%$, with an associated hospital mortality of 40 to $67 \%$. This study used the RIFLE criteria to analyze the effect of an immunosuppressive regime including calcineurin inhibitors $(\mathrm{CNi})$ in the development of $\mathrm{ARD}$ in patients with liver transplantation. It also assesses the protocol and progressive late introduction of $\mathrm{CNi}$ as a strategy to reduce the incidence of posttransplant ARD

Methods: cohort analytic study of 163 patients with liver transplantation

Results: 163 patients met the study inclusion criteria with an average age of 51 (46-56) years. Of these patients, only $11(6.74 \%)$ had ARD transplantation. Cyclosporine was administered to 126 $(77.3 \%)$ of tacrolimus in transplant patients and 21 (12.88\%). Immediately after the transplant, patients were classified into two groups: patients with high risk of developing ERA and patients without kidney problems. At first they were introduced to the CNI from the third day after transplantation and the last six to 18 hours after the transplant, a dose that was gradually increased

Conclusions: late and gradual introduction of $\mathrm{CNi}$ could be an effective strategy to reduce the incidence of acute renal disease in liver transplantation (Acta Med Colomb 2011; 36: 130-134).

Keywords: liver transplantation, acute renal disease, RIFLE criteria, calcineurin inhibitors

Dra. Yeinis Paola Espinoza Herrera: Estudiante de Medicina. Joven Investigadora del Grupo de Gastrohepatología. Universidad de Antioquia; Dr. Juan Carlos Restrepo Gutiérrez: Esp, MSc, PhD. Profesor Departamento de Medicina Interna. Grupo de Gastrohepatología. Universidad de Antioquia. Unidad de Hepatología y Trasplante de Hígado. Hospital Pablo Tobón Uribe; Dr. John Jairo Zuleta Tobón: Esp. Profesor, Grupo de Gastrohepatología. Universidad de Antioquia. Unidad de Epidemiología. Hospital Pablo Tobón Uribe; Dr. Juan Ignacio Marín Zuluaga: Esp. Residente de Hepatología. Grupo de Gastrohepatología. Universidad de Antioquia; Dr. Octavio Germán Muñoz Maya: Esp. Residente de Hepatología. Grupo de Gastrohepatología. Universidad de Antioquia; Dr. Sergio Iván Hoyos Duque: Esp. Profesor, Departamento de Cirugía. Grupo de Gastrohepatología. Universidad de Antioquia. Unidad de Hepatología y Trasplante de Hígado. Hospital Pablo Tobón Uribe; Dr. Carlos Guzmán Luna: Esp. Unidad de Hepatología y Trasplante de Hígado. Hospital Pablo Tobón Uribe; Dr. Álvaro Mena Hurtado: Esp. Unidad de Hepatología y Trasplante de Hígado. Hospital Pablo Tobón Uribe; Dr. Gonzalo Correa Arango: Esp. Profesor, Departamento de Medicina Interna. Grupo de Gastrohepatología. Universidad de Antioquia. Unidad de Hepatología y Trasplante de Hígado. Hospital Pablo Tobón Uribe. Medellín (Colombia).

Esta investigación se realizó en el Hospital Pablo Tobón Uribe, institución de carácter privado ubicada en la ciudad de Medellín, Colombia

Correspondencia. Dra. Yeinis Paola Espinoza Herrera. Grupo de Gastrohepatología. Universidad de Antioquia. Medellín (Colombia). E-mail: yeinis_eh@yahoo.com

Recibido: 01/XII/2010 Aceptado: 14/VII/2011 


\section{Introducción}

La enfermedad hepática terminal es un trastorno con una extensa lista de factores causales cuya única alternativa de tratamiento es el trasplante de hígado $(\mathrm{TH})(1,2)$. Una de las complicaciones más frecuentes de este procedimiento es el deterioro de la función renal que se presenta en el postrasplante inmediato con una incidencia que varía de $12-64 \%$, con una mortalidad intrahospitalaria asociada de $40-67 \%$ (3-7). Aunque estos datos dan cuenta de la importancia del tema, no hay consenso en cuanto al diagnóstico de la enfermedad renal aguda (ERA) en el postrasplante de hígado. Dos artículos publicados sobre el tema por Lima et al. y el de Cabezuelo et al reportan una incidencia de ERA a los 30 días postrasplante de 61 y $48 \%$, respectivamente. Sin embargo, otras investigaciones como la de Chuang et al., que utilizan una definición diferente para la ERA, reportan una incidencia de apenas $8 \%$ (8-10). Y mientras en dos estudios la aparición de ERA postrasplante se asoció a pobre pronóstico con elevada mortalidad postrasplante, en otras investigaciones la aparición de ERA postrasplante se relacionó con buen pronóstico $(4-6,11)$.

Por otro lado, la nefrotoxicidad inducida por los inhibidores de la calcineurina ( $\mathrm{CNi}$ ) se menciona en la mayoría de artículos como uno de los factores más importantes en la etiología de la ERA postrasplante (4,10-13). Dos estudios controlados evalúan la incidencia de ERA postrasplante de hígado: el de Barri et al y el de O'Riordan et al $(14,15)$. El primero utiliza diferentes definiciones para el diagnóstico de ERA postrasplante con el objetivo de determinar los niveles de creatinina que más se asocian a la aparición de ERA. El segundo utiliza los criterios RIFLE (Risk, Injury, Failure, Loss, End stage kidney disease) para el diagnóstico de este trastorno. De forma similar, diseñamos un estudio controlado en el que utilizando los criterios RIFLE analizamos el efecto del esquema inmunosupresor con $\mathrm{CNi}$ en la aparición de ERA en pacientes con TH y evaluamos el protocolo de introducción tardía y progresiva de los $\mathrm{CNi}$ como estrategia para disminuir la incidencia de este importante trastorno.

\section{Material y métodos}

Este estudio analítico de cohortes que contó para su realización con el aval del Comité de Ética del Hospital Pablo Tobón Uribe (HPTU), Medellín, Colombia, describe la incidencia de enfermedad renal aguda (ERA) en un grupo de 163 pacientes adultos mayores de 18 años a quienes se les realizó trasplante ortotópico de hígado $(\mathrm{TOH})$ entre el 12 de febrero de 2004 y el 9 de marzo de 2009, por el Grupo de Trasplante Hepático de la Universidad de Antioquia (U. de A.) y el HPTU.

Para el diagnóstico de ERA postrasplante se usó la clasificación RIFLE (Risk, Injury, Failure, Loss, End stage kidney disease) propuesta por la conferencia de consenso internacional del grupo ADQI (Acute Dialysis Quality Iniciative) en el año 2004, la cual define la ERA por un aumento de la creatinina al doble o al triple del valor pretrasplante dos semanas después del trasplante, o por una disminución de la tasa de filtración glomerular en 50 o $75 \%$ a las dos semanas postrasplante, de acuerdo con las fórmulas de Cockroft-Gault y a la ecuación de MDRD (Modification of Diet in Renal Disease) (Tabla 1) (16).

Se excluyeron los pacientes que tuvieron trasplante simultáneo de hígado y riñón ( $\mathrm{n}=3$ ), así como aquéllos con muerte o retrasplante antes de los quince días del primer TOH ( $\mathrm{n}=$ 17). Tampoco se incluyeron los datos de los pacientes que por alguna razón no recibieron algún inhibidor de la calcineurina ( $\mathrm{CNi}$ ) dentro del esquema de inmunosupresión de los primeros 15 días postrasplante.

La información fue obtenida de la base de datos del HPTU y complementada con la historia clínica electrónica de cada paciente. El Grupo de Trasplante Hepático de la U. de A. y el HPTU lleva un registro prospectivo de todos los pacientes trasplantados de hígado. Los pacientes se revisan regularmente según un protocolo preestablecido y estandarizado por parte del mismo grupo tratante. Los datos por estudiar fueron consignados en una base de datos diseñada en Excel, y posteriormente se analizaron con el paquete estadístico SPSS 15.0.

Varios parámetros fueron evaluados en asociación con la ERA. En el periodo pretrasplante: edad, sexo, enferme-

Tabla 1. Criterios RIFLE para el diagnóstico de enfermedad renal aguda.*

\begin{tabular}{|c|c|c|c|}
\hline RIFLE & Criterios de TFG & Criterios de diuresis & \\
\hline Risk (riesgo) & Aumento de creatinina sérica $\times 1.5$ o disminución de la TFG $\geq 25 \%$ & Diuresis $<0.5 \mathrm{~mL} / \mathrm{Kg} / \mathrm{h}$ por seis horas & \multirow{3}{*}{ Sensibilidad alta } \\
\hline Injury (Lesión) & Aumento de creatinina sérica $\mathrm{x} 2$ o disminución de la TFG $\geq 50 \%$ & Diuresis $<0.5 \mathrm{~mL} / \mathrm{Kg} / \mathrm{h}$ por 12 horas & \\
\hline Failure (Falla) & Aumento de creatinina sérica $x 3 \mathrm{o} \geq 4 \mathrm{mg} / \mathrm{dL}$ o disminución de la $\mathrm{TFG} \geq 75 \%$ & $\begin{array}{l}\text { Oliguria: diuresis }<0.3 \mathrm{~mL} / \mathrm{Kg} / \mathrm{h} \text { por } \\
24 \text { horas o anuria por } 12 \text { horas }\end{array}$ & \\
\hline Loss (Pérdida) & Pérdida completa de la función renal durante cuatro o más semanas & & \multirow[b]{2}{*}{ Especificidad alta } \\
\hline $\begin{array}{l}\text { End stage kidney } \\
\text { disease } \\
\text { (enfermedad renal } \\
\text { terminal) }\end{array}$ & Necesidad de diálisis ( $>3$ meses) & & \\
\hline
\end{tabular}


dad de base (definida como: cirrosis no biliar, cirrosis no biliar y carcinoma hepatocelular, falla hepática fulminante, colestasis crónica, tumor hepático en hígado sano, u otras) y puntaje tanto en la clasificación Child-Pugh como en la escala MELD (Model for End stage Liver Disease), según los parámetros descritos en la literatura $(17,18)$. Los antecedentes personales de hipertensión arterial, diabetes mellitus y enfermedad renal pretrasplante fueron descritos según reporte de diagnóstico o tratamiento en la historia clínica.

Los factores relacionados con el procedimiento quirúrgico que se tuvieron en cuenta fueron: las complicaciones perioperatorias (definidas como: trombosis de la arteria hepática, neuroconfusión, mielonisis póntica, falla cardiaca, hemoperitoneo, hemotórax masivo, ascitis postrasplante, infección del líquido ascítico, infección de la herida quirúrgica, sangrado masivo intraoperatorio, filtración de la vía biliar, mala función inicial, trombosis arterial, síndrome compartimental abdominal, bacteriemia, tromboembolismo pulmonar, ruptura de la vena cava y paro cardiorrespiratorio), el tiempo de isquemia fría (los minutos transcurridos desde el momento en que se pinza la aorta en el donante hasta que se aplica la solución de preservación) y el tiempo de isquemia caliente (los minutos transcurridos desde el momento en que se extrae el injerto de la nevera con la solución de preservación hasta la apertura de la vena porta en el receptor).

Finalmente, el factor postrasplante que se tuvo en cuenta en relación con la ERA fue la administración de algún CNi dentro del esquema de inmunosupresión de los pacientes.

Como parte del protocolo de inmunosupresión utilizado por el Grupo de Gastrohepatología a los pacientes con trasplante hepático se les administra durante el periodo intraoperatorio $1 \mathrm{~g}$ de metilprednisolona intravenosa. En el periodo posoperatorio inmediato los pacientes son clasificados en dos grupos: pacientes con insuficiencia renal o con riesgo elevado de desarrollarla y pacientes sin problemas renales actuales o potenciales. A los primeros se les administra o bien azatioprina a una dosis de $1-2 \mathrm{mg} / \mathrm{kg}$ o micofenolato $1000 \mathrm{mg} / 12$ horas, en los primeros tres a cinco días. Así mismo, metilprednisolona es administrada a las siguientes dosis desde el primer hasta el sexto día: $200 \mathrm{mg}$ el primer día, 160 el segundo, 120 el tercero, 80 el cuarto y $40 \mathrm{mg}$ el quinto día. A partir del sexto día se administra prednisolona a $20 \mathrm{mg} /$ día. En estos pacientes con insuficiencia renal o con riesgo alto de desarrollarla, el inhibidor de la calcineurina es administrado a partir del día tres o en el momento en que la función renal lo permita, mientras en aquellos pacientes sin problemas renales el inhibidor de la calcineurina es iniciado entre seis y dieciocho horas postrasplante a una dosis que se aumenta gradualmente hasta llegar al nivel objetivo en el primer mes: entre 800 y $1200 \mathrm{ng} / \mathrm{mL}$ en el caso de la ciclosporina.

Para el análisis, la cohorte fue dividida en dos grupos: pacientes que recibieron algún $\mathrm{CNi}$ antes de la presentación de la ERA y pacientes que nunca recibieron algún CNi durante todo el seguimiento, o que nunca habían recibido este tipo de medicamentos antes de presentar la ERA. Las variables cuantitativas, excepto la edad, no tuvieron una distribución normal según la prueba de Kolmogórov-Smirnov, por lo tanto se presentan como mediana y rango intercuartílico y las respectivas comparaciones entre los grupos se hizo mediante la prueba U de Mann Whitney. Se buscó la asociación con la ERA con las variables cualitativas mediante una prueba de chi cuadrado. Todas las comparaciones se consideraron significativas a un nivel de 0.05 .

\section{Resultados}

Durante el periodo comprendido entre febrero de 2004 y marzo de 2009, 196 pacientes recibieron trasplante de hígado (TH) en el HPTU, de los cuales, 163 cumplieron con los criterios de inclusión del estudio, siendo la mayoría de sexo masculino (63.8\%), con un promedio de edad de 51 (46-56) años (Tabla 2). Previo al TH, 26 pacientes (16\%) presentaban deterioro de la función renal, 34 (20.9\%) recibían tratamiento farmacológico para hipertensión arterial (HTA) y 32 (19.6\%) tenían antecedente personal de diabetes mellitus (DM).

A los quince días postrasplante sólo 11 pacientes (6.74\%) presentaron enfermedad renal aguda (ERA). Las características pretrasplante de estos pacientes y el impacto de estos factores en la función renal postrasplante de acuerdo con el análisis univariado se presentan en la Tabla 2. De estos 11 pacientes que presentaron ERA postrasplante ninguno recibía tratamiento para DM, dos (18.2\%) tenían antecedente personal de HTA pretrasplante, dos (18.2\%) tenían niveles elevados de creatinina previo al trasplante, siete (63.6\%) tenían enfermedad hepática estadio Child C, dos (18.2\%) Child B y ningún paciente con ERA postrasplante fue clasificado como Child A.

En cuanto a las indicaciones del TH: en siete (63.6\%) de los 11 pacientes que presentaron ERA postrasplante fue la cirrosis no biliar. Dos (18.2\%) fueron trasplantados con el diagnóstico de cirrosis no biliar y también dos (18.2\%) por falla hepática aguda (Tabla 2). Así mismo, cuatro (36.4\%) de estos pacientes con ERA postrasplante presentaron complicaciones perioperatorias y tres $(27.3 \%)$ requirieron reintervención postrasplante, dos de ellos por presentar complicaciones perioperatorias. Ninguno de los pacientes con ERA postrasplante presentó rechazo en los primeros quince días posterior al trasplante. La edad promedio de los donantes fallecidos fue de 36.18 ( $\mathrm{DS} \pm 12.83$ ) años y de 51 (DS \pm 12.19 ) años en los donantes cuyos receptores presentaron ERA postrasplante. Los valores de isquemia caliente y de isquemia fría fueron muy similares en los pacientes que presentaron ERA postrasplante y en quienes la función renal no se alteró en el postrasplante inmediato, y no hubo diferencias significativas al comparar estos resultados en ambos grupos (Tabla 2).

La ciclosporina fue el CNi más usado, pues se administró a $126(77.3 \%)$ de los pacientes trasplantados en algún momento de los 15 días postrasplante. Tacrolimus, por su parte 
Tabla 2. Características clínicas de los pacientes del estudio.

\begin{tabular}{|c|c|c|c|}
\hline Característica & $\begin{array}{l}\text { Con ERA } \\
\text { (11) }\end{array}$ & $\begin{array}{l}\text { Sin ERA } \\
\quad(152)\end{array}$ & $\mathbf{p}$ \\
\hline Sexo (M:F) & $8: 3(72.7 \%: 27.3 \%)$ & $96: 56(63.2 \%: 36.8 \%)$ & 0.387 \\
\hline \multicolumn{4}{|l|}{ Pretrasplante } \\
\hline Edad & $51(46-56)$ & $54(42-60)$ & 0.578 \\
\hline Hipertensión arterial & $2(18.2 \%)$ & $32(21.1 \%)$ & 1 \\
\hline Diabetes mellitus & $0(0 \%)$ & $32(21.1 \%)$ & 0.124 \\
\hline Enfermedad renal & $2(18.2 \%)$ & $24(15.8 \%)$ & 0.689 \\
\hline Indicación del trasplante & & & 0.576 \\
\hline Cirrosis no biliar & $7(63.6 \%)$ & $111(73 \%)$ & \\
\hline Colestasis crónica & $2(18.2 \%)$ & $20(13.2 \%)$ & \\
\hline Falla hepática aguda & $2(18.2 \%)$ & $7(4.6 \%)$ & \\
\hline Cirrosis no biliar y CHC & $0(0 \%)$ & $3(2 \%)$ & \\
\hline $\begin{array}{l}\text { Tumor hepático } \\
\text { en hígado sano }\end{array}$ & $0(0 \%)$ & $2(1.3 \%)$ & \\
\hline Retrasplante & $0(0 \%)$ & $4(2.6 \%)$ & \\
\hline Otras & $0(0 \%)$ & $5(3.3 \%)$ & \\
\hline Estadio Child & & & 0.295 \\
\hline A & $0(0 \%)$ & $16(10.5 \%)$ & \\
\hline B & $2(18.2 \%)$ & $53(34.9 \%)$ & \\
\hline $\mathrm{C}$ & $7(63.6 \%)$ & $70(46.1 \%)$ & \\
\hline Sin registro & $2(18.2 \%)$ & $13(8.6 \%)$ & \\
\hline Perioperatorio & & & \\
\hline $\begin{array}{l}\text { Isquemia fría } \\
\text { (tiempo en minutos) }\end{array}$ & $355(300-345)$ & $330(291-420)$ & 0.474 \\
\hline $\begin{array}{l}\text { Isquemia caliente } \\
\text { (tiempo en minutos) }\end{array}$ & $22(20-35)$ & $27(23-32)$ & 0.296 \\
\hline Complicaciones & & & 0.733 \\
\hline Sí & $4(36.4 \%)$ & $44(28.9 \%)$ & \\
\hline No & $7(63.6 \%)$ & $108(71.1 \%)$ & \\
\hline Reintervención & & & 0.427 \\
\hline Sí & $3(27.3 \%)$ & $27(17.8 \%)$ & \\
\hline No & $8(72.7 \%)$ & $125(82.2 \%)$ & \\
\hline Periodo postrasplante & & & \\
\hline Uso de ciclosporina & $9(81.8 \%)$ & $119(78.3 \%)$ & 0.273 \\
\hline Uso de tacrolimus & $2(18.2 \%)$ & $19(12.5 \%)$ & 0.636 \\
\hline
\end{tabular}

fue usado en 21 (12.88\%) pacientes (Tabla 2). En ningún caso se administró tratamiento secuencial con ciclosporina y tacrolimus. Micofenolato y azatioprina siempre fueron administrados junto a un esteroide, $\mathrm{y}$ en todos los casos el CNi se adicionó o bien a la combinación de esteroide y azatioprina, o bien a la de esteroide y micofenolato. Al momento de presentar la ERA postrasplante nueve (81.8\%) de los 11 pacientes recibían tratamiento con ciclosporina y dos $(18.2 \%)$ venían recibiendo tacrolimus.
Cuatro $(36.4 \%)$ de los 11 pacientes con ERA postrasplante aún viven, cuatro (36.4\%) murieron antes de completar tres meses postrasplante y desconocemos si tres (27.3\%) de ellos aún viven, pues residen fuera del país. En seis $(54.5 \%)$ de los 11 casos con ERA postrasplante la función renal mejoró tras la disminución de la dosis del inhibidor de la calcineurina y a cinco (36.4\%) pacientes se les suspendió el inhibidor de la calcineurina y les fue administrado temporalmente tratamiento sólo con esteroide junto a micofenolato.

\section{Discusión}

Utilizando los criterios RIFLE encontramos una muy baja incidencia de enfermedad renal aguda (ERA) postrasplante sólo $11(6.74 \%)$ de los 163 pacientes incluidos. Estos datos están muy por debajo de lo descrito en otros estudios (8-10). O'Riordan et al también utilizan los criterios RIFLE para evaluar la incidencia de ERA postrasplante. Ellos reportan una incidencia de ERA de $25.7 \%$ (15).

Diferentes investigaciones han planteado estrategias para disminuir la incidencia de disfunción renal postrasplante en pacientes con factores de riesgo: Barri y cols. proponen en su estudio iniciar el esquema de inmunosupresión con esteroides y micofenolato, y diferir la introducción de la ciclosporina hasta después de las primeras 72 horas postrasplante, o en los pacientes con ERA, cuando mejoren los niveles bioquímicos de función renal (19). Nosotros utilizamos una estrategia similar a ésta, de modo que los pacientes trasplantados de hígado recibían inmunosupresión con esteroide y micofenolato o azatioprina; mientras el inhibidor de la calcineurina $(\mathrm{CNi})$ era introducido en dosis crecientes y de forma progresiva. Es así como la baja incidencia de ERA postrasplante que reportamos puede deberse a esta estrategia de inmunosupresión.

Teniendo en cuenta que la nefrotoxicidad de los $\mathrm{CNi}$ depende de la dosis, otra estrategia empleada ha sido la disminución de la dosis administrada. Y aunque esto no previene la aparición de ERA postrasplante, sí disminuye la morbilidad y mortalidad asociadas a este importante trastorno. Varo y cols. observaron en su estudio mejoría de la función renal tras iniciar un esquema de inmunosupresión que incluía la administración de dosis bajas y crecientes de tacrolimus en aquellos pacientes con deterioro de la función renal en el postrasplante inmediato (20). Así mismo, nuestro estudio reporta una baja prevalencia de ERA postrasplante tras la disminución de la dosis de $\mathrm{CNi}$ a aquellos pacientes que presentaban alteración en los niveles de creatinina en el postrasplante inmediato.

Entre los otros protocolos utilizados para disminuir la incidencia de ERA en pacientes de alto riesgo se encuentra la introducción tardía de la ciclosporina mientras se administra micofenolato en combinación con los anticuerpos monoclonales basiliximab o daclizumab, y la administración de un esquema de inmunosupresión sin ciclosporina (21). En nuestra cohorte de pacientes trasplantados siempre uti- 
lizamos algún CNi dentro del esquema de inmunosupresión postrasplante.

Entre las limitaciones del presente estudio debemos considerar que podría haber un sesgo derivado de la recolección de los datos por ser un estudio retrospectivo. Diferentes investigaciones han reportado los antecedentes personales de hipertensión arterial, diabetes mellitus y deterioro renal pretrasplante; así como la aparición de complicaciones perioperatorias y la necesidad de reintervención, como factores de riesgo para ERA postrasplante $(4,8,10,15)$. Sin embargo, en nuestro estudio, pese a encontrar estas diferencias, no alcanzaron significancia estadística, lo que posiblemente se debe al tamaño de la muestra.

En conclusión, la baja incidencia de ERA que reportamos en el postrasplante de hígado muy probablemente se debe a una combinación de diferentes estrategias de inmunosupresión. Esta estrategia se resume en la introducción tardía de los inhibidores de la calcineurina a dosis crecientes y según los resultados de las pruebas de función renal. Finalmente recomendamos el uso de los criterios RIFLE en estudios posteriores, ya que esto permitirá comparar los resultados entre investigaciones realizadas en diferentes grupos de pacientes trasplantados

\section{Referencias}

1. Randhawa $\mathbf{P}$, Shapiro R. Chronic renal failure after liver transplantation. Am J Transplant 2005; 5: 967-69.

2. Ling Q, Xu X, Li J, Chen J, Shen J, Zheng S. Alternative Definition of Acute Kidney Injury Following Liver Transplantation: Based on serum creatinine and Cystatin C Levels. Transplant Proc 2007; 39: 3257-60.

3. Lebron M, Herrera M, Seller G. Risk factors for renal dysfunction in the postoperative course of liver transplant. Liver Transplant 2004; 10:1379-81.

4. Chuang $\mathbf{F}$, Lin $\mathbf{C}$, Wang $\mathbf{P}$, Cheng Y, Hsu K, Chen Y, et al. Acute renal failure after cadaveric related liver transplantation. Transplant Proc 2004; 36: 2328-30.

5. Wei Y, Zhang $\mathbf{L}, \mathbf{L i n} \mathbf{H}, \mathbf{L i} \mathbf{J}, \mathbf{L i} B$, Yan $\mathbf{L}$, et al. Factors related to post-liver transplantation acute renal failure. Transplant Proc 2006; 38: 2982-4.

6. Faenza S, Santoro A, Mancini E, Pareschi S, Siniscalchi A, Zanzani C, et al. Acute renal failure requiring renal replacement therapy after orthotopic liver transplantation. Transplant Proc 2006; 38: 1141-2.

7. Cabezuelo J, Ramirez P, Acosta F, Sanchez F, Robles R, Pons J, et al. Prognostic factors of early acute renal failure in liver transplantation. Liver Transplant 2002; 34 (1): 254-5

8. Lima E, Dirce M, Castro I, Massarollo P, Miles S, Machado M, et al. Risk factors for development of acute renal failure after liver transplantation. Ren Fail 2005; 4: 553-60.

9. Cabezuelo J, Ramírez J, Ríos A, Acosta F, Torres D, Sansano T, et al. Risk factors of acute renal failure after liver transplantation. Kidney Int 2006; 69: 1073-80.

10. Chuang F, Lee C, Chien Y, Chen Y, Chen C. Acute renal failure after livingrelated liver transplantation. Transplant Proc 2003; 35: 429-30.

11. Junge G, Schewior L, Kohler S, Neuhaus R, Langrehr J, Tullius S, et al. Acute renal failure after liver transplantation: incidence, etiology, therapy, and outcome. Transplant Proc 2006; 38: 723-4.

12.Paydas S, Balal M, Demiryurek H, Kose F. Renal function in patients with orthotopic liver transplantation. Ren Fail 2006; 28: 103-5.

13. Gonwa T, Mai M, Melton L, Hays S, Goldstein R, Levy M, et al. End-stage rena disease (ESRD) after orthotopic liver transplantation (OLTX) using calcineurinbased immunotherapy. Transplantation 2001; 72: 1934-9.

14. Barri Y, Sanchez E, Jennings L, Melton L, Hays S, Levy M, Klintmalm G. Acute Kidney Injury Following Liver Transplantation: Definition and Outcome. Liver Transplant 2009; 15: 475-83.

15. O'Riordan A, Wong V, McQuillan R, McCormick P, Hegarty J, Watson A. Acute Renal Disease, as defined by the RIFLE Criteria, Post-Liver Transplantation. Am J Transplant 2007; 7: 168-76.

16. Bellomo R, Ronco C, Kellum JA, Mehta RL, Palevsky P. Acute renal failure-definition, outcome measures, animal models, fluid therapy and information technology needs: The Second International Consensus Conference of the Acute Dialysis Quality Initiative (ADQI) Group. Crit Care 2004; 8: R204-12.

17. Child C, Turcotte J. The liver and portal hypertension. $1^{\text {a }}$ ed. Philadelphia: Saunders; 1964

18. Malinchoc M, Kamath P, Gordon F, Peine C, Rank P, Borg P. A model to predict poor survival in patients undergoing transjugular intrahepatic portosystemic shunts. Hepatology 2000; 31: 864-71.

19. Barri Y, Sanchez E, Jennings L, Melton L, Hays S, Levy M, Klintmalm G. Acute Kidney Injury Following Liver Transplantation: Definition and Outcome. Liver Transplant 2009; 15: 475-83.

20. Varo E, Lopez A, Castroagudin J.Alternative immunosuppression for acute renal failure in liver transplantation: role of ultra-low dose tacrolimus and basiliximab. Transplant Proc 2002; 34: 1533-4.

21. Varo E, Lopez A, Rivero C. Initial immunosuppression in liver transplant recipients with impaired renal function. Transplant Proc 2005; 37: 3909-12. 